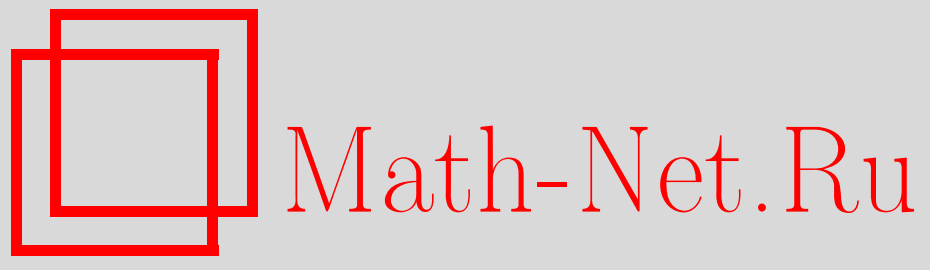

S. Hamadene, Y. Ouknine, Reflected backward SDEs with general jumps, Теория вероятн. и ее примен., 2015, том 60, выпуск $2,357-376$

DOI: https://doi.org/10.4213/tvp4623

Использование Общероссийского математического портала Math-Net.Ru подразумевает, что вы прочитали и согласны с пользовательским соглашением http: //www . mathnet.ru/rus/agreement

Параметры загрузки:

IP: 3.82 .47 .9

26 апреля 2023 г., 16:22:35

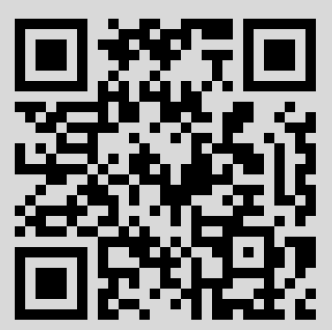




\title{
REFLECTED BACKWARD SDES WITH GENERAL JUMPS
}

\begin{abstract}
Изучаются одномерные обратные стохастические дифференциальные уравнения (ОСДУ) с отражением, управляемые броуновским движением и независимой пуассоновской случайной мерой, в случае, когда генератор липшицев. Сначала мы доказываем существование и единственность решения ОСДУ с одной отражающей границей, когда процесс-препятствие непрерывен справа, имеет пределы слева и обладает произвольной структурой скачков. Затем, основываясь на условии Мокободски, мы устанавливаем существование и единственность решения ОСДУ с отражением в случае двух отражающих границ, непрерывных справа и имеющих пределы слева.
\end{abstract}

Ключевые слова и фразы: обратные стохастические дифференциальные уравнения, штраф, пуассоновский точечный процесс, оболочка Снелла, гипотеза Мокободски.

1. Introduction. The class of backward stochastic differential equations (BSDEs for short) driven by a Brownian motion $\left(B_{t}\right)_{t \leqslant T}$ was introduced by Pardoux and Peng [16]. A solution of such a BSDE is a pair of processes $\left(Y_{t}, Z_{t}\right)_{t \leqslant T}$ that satisfies

$$
Y_{t}=\xi+\int_{t}^{T} f\left(s, \omega, Y_{s}, Z_{s}\right) d s-\int_{t}^{T} Z_{s} d B_{s}, \quad t \leqslant T,
$$

where $\xi$ and $f$ are given. Their aim was to give a probabilistic interpretation of a solution of a second order quasi-linear partial differential equation. Since then, these equations gradually became a very useful tool in several fields of mathematics such as finance, optimal stochastic control, differential games, and nonlinear partial differential equations to mention a few (see, e.g., [1]-[3], [6]-[8], [10], [14], [17], [18] and the references therein).

In connection with optimal stochastic control, Tang and Li [14] considered BSDEs driven by a Brownian motion $B$ and an independent Poisson

${ }^{*}$ Université du Maine, Laboratoire Manceau de Mathématiques, Le Mans, France; e-mail: hamadene@univ-lemans.fr

**Département de Mathématiques, Faculté des Sciences Semlalia, Université Cadi Ayyad, Marrakech, Maroc; e-mail: ouknine@ucam.ac.ma 
random measure $\mu$. In this setting a solution of such a BSDE is a triple of processes $\left(Y_{t}, Z_{t}, V_{t}\right)_{t \leqslant T}$ that satisfies: for all $t \leqslant T$,

$$
Y_{t}=\xi+\int_{t}^{T} f\left(s, \omega, Y_{s}, Z_{s}\right) d s-\int_{t}^{T} Z_{s} d B_{s}-\int_{t}^{T} \int_{\mathbf{R}^{\ell} \backslash\{0\}} V_{s}(e) \tilde{\mu}(d s, d e),
$$

where $\widetilde{\mu}$ is the compensated measure associated with $\mu$. As in the seminal work by Pardoux-Peng [16], they proved existence and uniqueness of the solution when $f$ is Lipschitz in $(y, z, v)$ and $\xi$ is square integrable. The link between this class of BSDEs and viscosity solutions of integral-partial differential equations is studied in [1].

Reflected BSDEs with one reflecting barrier driven by a Brownian noise have been considered first in [6]. In this framework the component $Y$ of the solution is forced to stay above a given stochastic obstacle process $\left(S_{t}\right)_{t \leqslant T}$, i.e., to satisfy $Y_{t} \geqslant S_{t}$ for any $t \leqslant T$. A solution of such a reflected BSDE is a triple of processes $\left(Y_{t}, Z_{t}, K_{t}\right)_{t \leqslant T}$, where $K$ is a nondecreasing continuous process which insures the condition $Y \geqslant S$ to be satisfied. Hamadène [9] extended the results of [6] to the wider class of barrier processes which are right continuous with left limits. This type of BSDEs turn out very useful in pricing of American options in constrained markets [7], among other obstacle problems. The generalization of the setting considered in [6] to the case of two reflecting barriers was carried out first by Cvitanic-Karatzas [3].

Later on, we considered in [11] reflected BSDEs with one reflecting barrier when the noise is driven by a Brownian motion and an independent Poisson measure. We showed existence and uniqueness of the solution when the barrier process has only inaccessible jumps, i.e., jumps which are related to the Poisson random measure $\mu$.

The main objective of this paper is to study reflected BSDEs when the noise is driven by a Brownian motion and an independent Poisson random measure, whereas the barrier processes are only right continuous with left limits. No further conditions are required on the nature of their jumps. The jumps can in fact be predictable or inaccessible. We consider the case of one reflecting barrier as well as the case of two reflecting barriers. For both cases we show existence and uniqueness of the solution when the coefficients of the BSDEs are Lipschitz. This class of BSDEs with discontinuous barrier processes seem play an important role in the pricing and risk management of convertible bonds in defaultable markets (see, e.g., [2]). We also believe that the main results of our work open a way towards viscosity solutions of variational inequalities with discontinuous obstacles.

This paper is organized as follows. In Section 2 we formulate the problem and set up the main assumptions. Section 3 is devoted to the proof of uniqueness of the solution. In Section 4, we address the question of existence of the solution of the reflected BSDEs with one reflecting barrier. We first deal with the case when $f$ does not depend on $(y, z, v)$. This is mainly due to 
the fact that comparison of solutions of standard BSDEs of type (2) may fail when the generators depend on $v$. Our method combines penalization arguments with the general theory of Snell envelope of processes. Furthermore, in order to obtain the result for generators depending on $(y, z, v)$, we introduce a contraction mapping in an appropriate Banach space of processes which has a fixed point, which in turn provides the unique solution of the BSDE. We end this section by investigating some properties of the reflecting process $K$ of the solution. In Section 5, we address the problem of reflected BSDEs with two reflecting barriers. Under the well-known Mokobodski hypothesis, we show existence and uniqueness of the solution. Finally Section 6 contains an appendix on the Snell envelope notion and optimal stopping.

2. Setting of the problem and assumptions. We first fix the horizon $T$ of the problem equal to 1 , the results still valid if $T$ differs from 1 . Let $\left(\Omega, \mathscr{F},\left(\mathscr{F}_{t}\right)_{t \leqslant 1}, \mathbf{P}\right)$ be a stochastic basis such that $\mathscr{F}_{0}$ contains all $\mathbf{P}$-null sets of $\mathscr{F}$ and $\mathscr{F}_{t+}=\bigcap_{\varepsilon>0} \mathscr{F}_{t+\varepsilon}=\mathscr{F}_{t}$ for any $t<1$. We assume that $\left(\mathscr{F}_{t}\right)_{t \leqslant 1}$ is generated by the two following mutually independent processes:

(i) a $d$-dimensional Brownian motion $\left(B_{t}\right)_{t \leqslant 1}$;

(ii) a random Poisson measure $\mu$ on $\mathbf{R}_{+} \times \mathbf{U}$, where $\mathbf{U}:=\mathbf{R}^{\ell} \backslash\{0\}$ is equipped with its Borel $\sigma$-algebra $\mathscr{U}$, with compensator $\nu(d t, d e)=d t \lambda(d e)$, such that $\{\tilde{\mu}([0, t] \times A)=(\mu-\nu)([0, t] \times A)\}_{t \leqslant 1}$ is a martingale for every $A \in \mathscr{U}$ satisfying $\lambda(A)<\infty$. The measure $\lambda$ is assumed to be $\sigma$-finite on $(\mathbf{U}, \mathscr{U})$ and integrates the function $U \ni e \mapsto 1 \wedge|e|^{2}$.

Next introduce the followings notation:

- $\mathrm{L}^{2}(\mathbf{U}, d \lambda)$ is the space of deterministic Borel functions $\varpi:=$ $(\varpi(e))_{e \in \mathbf{U}}$ from $\mathbf{U}$ to $\mathbf{R}$ which are $d \lambda$-square integrable, i.e., $\|\varpi\|=$ $\left(\int_{\mathbf{U}}|\varpi(e)|^{2} d \lambda\right)^{1 / 2}<\infty$;

- $\mathscr{S}^{2}$ is the set of $\mathscr{F}_{t}$-adapted RCLL processes $Y:=\left(Y_{t}\right)_{t \leqslant 1}$ with values in $\mathbf{R}$ and $\mathbf{E}\left[\sup _{t \leqslant 1}\left|Y_{t}\right|^{2}\right]<\infty$; we denote by $\mathscr{S}_{i}^{2}$ the subset of $\mathscr{S}^{2}$ which contains nondecreasing processes $K:=\left(K_{t}\right)_{t \leqslant 1}$ with $K_{0}=0$;

- $\mathscr{P}$ is the $\sigma$-algebra of $\mathscr{F}_{t}$-progressively measurable sets on $\Omega \times[0,1]$;

- $\mathscr{H}^{2, k}$ is the set of $\mathscr{P}$-measurable processes $Z:=\left(Z_{t}\right)_{t \leqslant 1}$ with values in $\mathbf{R}^{k}$ and $d \mathbf{P} \otimes d t$-square integrable;

- $\mathscr{P}_{d}$ is the $\sigma$-algebra of $\mathscr{F}_{t}$-predictable sets on $\Omega \times[0,1]$;

- $\mathscr{L}^{2}$ is the set of mappings $V: \Omega \times[0,1] \times \mathbf{U} \rightarrow \mathbf{R}$, which are $\mathscr{P}_{d} \otimes \mathscr{U}$ measurable and $d \mathbf{P} \otimes d t \otimes d \lambda$-square integrable; P-a.s.;

- for $s \leqslant 1, \mathscr{T}_{s}$ is the set of $\mathscr{F}_{t}$-stopping times $\tau$ such that $s \leqslant \tau \leqslant 1$,

- the class $[\mathrm{D}]$ is the set of $\mathscr{F}_{t}$-adapted RCLL processes $\zeta=\left(\zeta_{t}\right)_{t \leqslant 1}$ such that the set of random variables $\left\{\zeta_{\tau}, \tau \in \mathscr{T}\right\}$ is uniformly integrable;

- for a given RCLL process $\left(J_{t}\right)_{t \leqslant 1}$ and for any $t \leqslant 1$, we define

$$
J_{t-}=\lim _{s \nearrow t} J_{s} \quad\left(J_{0-}=J_{0}\right), \quad \Delta_{t} J=J_{t}-J_{t-}, \quad J_{-}:=\left(J_{t-}\right)_{t \leqslant 1}
$$


We are now given three objects which satisfy the following assumptions:

(a) a terminal value $\xi \in L^{2}\left(\Omega, \mathscr{F}_{1}, \mathbf{P}\right)$;

(b) a map $f: \Omega \times[0,1] \times \mathbf{R}^{1+d} \times \mathrm{L}^{2}(\mathbf{U}, d \lambda) \rightarrow \mathbf{R}$ which with $(t, \omega, y, z, v)$ associates $f(t, \omega, y, z, v)$ and which is $\mathscr{P} \otimes \mathscr{B}\left(\mathbf{R}^{1+d}\right) \otimes \mathscr{B}\left(\mathrm{L}^{2}(\mathbf{U}, d \lambda)\right)$ measurable; in addition we assume that:

(i) the process $(f(t, 0,0,0))_{t \leqslant 1}$ belongs to $\mathscr{H}^{2,1}$;

(ii) $f$ is uniformly Lipschitz with respect to $(y, z, v)$, i.e., there exists a constant $C_{f} \geqslant 0$ such that for any $(y, z),\left(y^{\prime}, z^{\prime}\right) \in \mathbf{R}^{1+d}$ and $v, v^{\prime} \in \mathrm{L}^{2}(\mathbf{U}, d \lambda)$ we have $\mathbf{P}$-a.s.,

$$
\left|f(\omega, t, y, z, v)-f\left(\omega, t, y^{\prime}, z^{\prime}, v^{\prime}\right)\right| \leqslant C_{f}\left(\left|y-y^{\prime}\right|+\left|z-z^{\prime}\right|+\left\|v-v^{\prime}\right\|\right) ;
$$

(c) an obstacle process $S:=\left(S_{t}\right)_{t \leqslant 1}$ which belongs to $\mathscr{S}^{2}$.

Let us now introduce the reflected BSDE with general jumps associated with the triple $(f, \xi, S)$. A solution is a quadruple $(Y, Z, K, V):=$ $\left(Y_{t}, Z_{t}, K_{t}, V_{t}\right)_{t \leqslant 1}$ of processes with values in $\mathbf{R}^{1+d} \times \mathbf{R}^{+} \times \mathrm{L}^{2}(\mathbf{U}, d \lambda)$ such that:

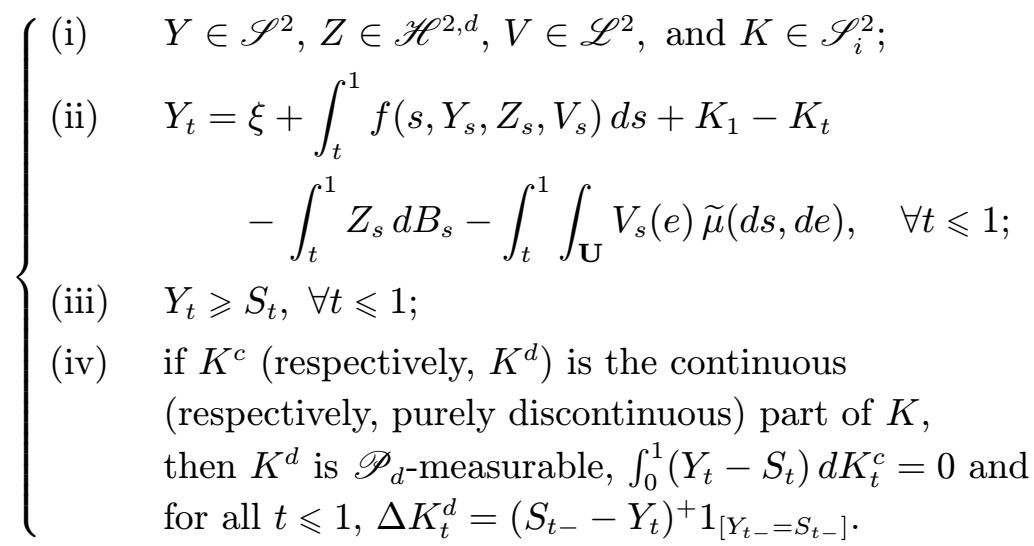

The process $Y$ has two types of jumps. The inaccessible ones which stem from the martingale $\left(\int_{0}^{t} \int_{\mathbf{U}} V_{s}(e) \tilde{\mu}(d s, d e)\right)_{t \leqslant 1}$ and the predictable ones which come from the predictable negative jumps of $S$. Those latter are the source of the predictable jumps of $Y$ and then also of $K$, which of course are the same. Thus the condition $\Delta_{t} K^{d}=\left(S_{t-}-Y_{t}\right)^{+} 1_{\left[Y_{t-}=S_{t-}\right]}$ is just a characterization of the predictable jumps of $Y$.

$\mathrm{R}$ e $\mathrm{m}$ a $\mathrm{r} \mathrm{k}$ 2.1. The second part of condition (iv) implies in particular that

$$
\int_{0}^{1}\left(Y_{t-}-S_{t-}\right) d K_{t}=0 .
$$

Actually

$$
\begin{aligned}
\int_{0}^{1}\left(Y_{t-}-S_{t-}\right) d K_{t} & =\int_{0}^{1}\left(Y_{t-}-S_{t-}\right) d K_{t}^{c}+\int_{0}^{1}\left(Y_{t-}-S_{t-}\right) d K_{t}^{d} \\
& =\int_{0}^{1}\left(Y_{t}-S_{t}\right) d K_{t}^{c}+\sum_{t \leqslant 1}\left(Y_{t-}-S_{t-}\right) \Delta K_{t}^{d}=0 .
\end{aligned}
$$


Here the sum $\sum_{t \leqslant 1}$ equals to 0 , since $K^{d}$ jumps only when $Y_{t-}=S_{t-}$.

Next we are going to focus on the uniqueness of the solution of the $\operatorname{BSDE}(3)$.

\section{Uniqueness of the solution of (3).}

Proposition 3.1. Under Assumptions (a)-(c) on $\left(f, \xi,\left(S_{t}\right)_{t \leqslant 1}\right)$, the associated reflected BSDE (3) has at most one solution.

$\mathrm{P}$ r o o f. Let us consider two solutions $(Y, Z, K, V)$ and $\left(Y^{\prime}, Z^{\prime}, K^{\prime}, V^{\prime}\right)$ of (3). First assume that

$$
\forall t \leqslant 1, \quad \int_{t}^{1}\left(Y_{s-}-Y_{s-}^{\prime}\right)\left(d K_{s}-d K_{s}^{\prime}\right) \leqslant 0
$$

For $t \leqslant 1$ we set

$$
\Delta_{t}:=\left|Y_{t}-Y_{t}^{\prime}\right|^{2}+\int_{t}^{1}\left|Z_{s}-Z_{s}^{\prime}\right|^{2} d s+\int_{t}^{1} \int_{\mathbf{U}}\left(V_{s}(e)-V_{s}^{\prime}(e)\right)^{2} \lambda(d e) d s .
$$

Then applying Itô's formula with $\left(Y-Y^{\prime}\right)^{2}$ and taking expectation yield

$$
\mathbf{E}\left[\Delta_{t}\right] \leqslant 2 \int_{t}^{1} \mathbf{E}\left[\left(Y_{s}-Y_{s}^{\prime}\right)\left(f\left(s, Y_{s}, Z_{s}, V_{s}\right)-f\left(s, Y_{s}^{\prime}, Z_{s}^{\prime}, V_{s}^{\prime}\right)\right)\right] d s
$$

for all $t \leqslant 1$. Therefore due to the inequality $2|a b| \leqslant 2 a^{2}+b^{2} / 2, a, b \in \mathbf{R}$, and since $f$ is uniformly Lipschitz with respect to $(y, z, v)$, we obtain

$$
\begin{gathered}
\mathbf{E}\left[\left|Y_{t}-Y_{t}^{\prime}\right|^{2}+\frac{1}{2} \int_{t}^{1}\left|Z_{s}-Z_{s}^{\prime}\right|^{2} d s+\frac{1}{2} \int_{t}^{1} \int_{\mathbf{U}}\left(V_{s}(e)-V_{s}^{\prime}(e)\right)^{2} \lambda(d e) d s\right] \\
\leqslant\left(2 C_{f}+4 C_{f}^{2}\right) \mathbf{E}\left[\int_{t}^{1}\left(Y_{s}-Y_{s}^{\prime}\right)^{2} d s\right], \quad \forall t \leqslant 1
\end{gathered}
$$

Then by Gronwall's lemma and the right continuity of $Y-Y^{\prime}$, we get $Y=Y^{\prime}$. It follows from $(6)$ that $(Z, V)=\left(Z^{\prime}, V^{\prime}\right)$ and finally $K=K^{\prime}$. Thus the solution of $(3)$ is unique. To complete the proof it remains to show that

$$
\int_{] t, 1]}\left(Y_{s-}-Y_{s-}^{\prime}\right)\left(d K_{s}-d K_{s}^{\prime}\right) \leqslant 0 \quad \forall t \leqslant 1 .
$$

Actually for any $t \leqslant 1$ we have

$$
\begin{aligned}
& \int_{] t, 1]}\left(Y_{s-}-Y_{s-}^{\prime}\right)\left(d K_{s}-d K_{s}^{\prime}\right) \\
& \quad=\int_{] t, 1]}\left(Y_{s-}-Y_{s-}^{\prime}\right)\left(d K_{s}^{c}-d K_{s}^{\prime c}\right)+\int_{] t, 1]}\left(Y_{s-}-Y_{s-}^{\prime}\right)\left(d K_{s}^{d}-d K_{s}^{\prime d}\right) .
\end{aligned}
$$


But $Y$ and $Y^{\prime}$ belong to $\mathscr{S}^{2}$ and their jumps $\delta(\omega):=\left\{t \in[0,1], \Delta_{t} Y(\omega) \neq 0\right\}$ and $\delta^{\prime}(\omega):=\left\{t \in[0,1], \Delta_{t} Y^{\prime}(\omega) \neq 0\right\}$ are at most countable, therefore

$$
\begin{aligned}
\int_{] t, 1]} & \left(Y_{s-}-Y_{s-}^{\prime}\right)\left(d K_{s}^{c}-d K_{s}^{\prime c}\right)=\int_{] t, 1]}\left(Y_{s}-Y_{s}^{\prime}\right)\left(d K_{s}^{c}-d K_{s}^{\prime c}\right) \\
& =\int_{] t, 1]}\left(Y_{s}-S_{s}\right)\left(d K_{s}^{c}-d K_{s}^{\prime c}\right)+\int_{] t, 1]}\left(S_{s}-Y_{s}^{\prime}\right)\left(d K_{s}^{c}-d K_{s}^{\prime c}\right) \\
& =-\int_{] t, 1]}\left(Y_{s}-S_{s}\right) d K_{s}^{\prime c}+\int_{] t, 1]}\left(S_{s}-Y_{s}^{\prime}\right) d K_{s}^{c} \leqslant 0 .
\end{aligned}
$$

On the other hand we have

$$
\begin{aligned}
\int_{] t, 1]} & \left(Y_{s-}-Y_{s-}^{\prime}\right)\left(d K_{s}^{d}-d K_{s}^{\prime d}\right) \\
& =\int_{] t, 1]}\left(Y_{s-}-Y_{s-}^{\prime}\right) d K_{s}^{d}-\int_{] t, 1]}\left(Y_{s-}-Y_{s-}^{\prime}\right) d K_{s}^{\prime d} .
\end{aligned}
$$

But $\int_{] t, 1]}\left(Y_{s-}-Y_{s-}^{\prime}\right) d K_{s}^{\prime d}=\int_{] t, 1]}\left(Y_{s-}-S_{s-}\right) d K_{s}^{\prime d} \geqslant 0$, since the jumps of $K^{\prime d}$ occur only when $Y_{-}^{\prime}=S_{-}$. Similarly we have $\int_{] t, 1]}\left(Y_{s-}-Y_{s-}^{\prime}\right) d K_{s}^{d} \leqslant 0$. Then the left-hand side of $(9)$ is $\leqslant 0$, and taking into account (8) leads to (7). The proof of uniqueness of the solution of the BSDE (3) is then complete.

\section{Existence of the solution.}

4.1. Preliminary case when $f$ does not depend on $(y, z, v)$ and penalization method. We are now going to prove that equation (3) has a solution. Our method combines penalization with the Snell envelope methods. Penalization as it has been used, e.g., in [6] cannot be applied because of lack of comparison of the solutions of the penalization scheme. To get this comparison we are led to assume, in a first step, that the function $f(t, \omega, y, z, v)$ does not depend on $v$ and for the sake of simplicity we will suppose moreover that $f$ does not depend also on $(y, z)$, i.e., $f(t, \omega, y, z, v)=g(t, \omega)$. Later on to obtain the result in the general setting we will use a fixed point argument with an appropriate mapping.

Note that as a by-product of the penalization method, we get the approximation of the solution of the reflected equation by solutions of BSDEs without reflection.

Assume that $\mathbf{P}$-a.s., for any $(t, y, z, v)$, we have $f(t, \omega, y, z, v)=g(t, \omega)$, where the process $(g(t, \omega))_{t \leqslant 1}$ belongs to $\mathscr{H}^{2,1}$. Then we are going to prove existence of a solution of the standard BSDE associated with $(g, \xi, S)$, by using the penalization method.

Theorem 4.1. The one barrier reflected BSDE (3) associated with $(g, \xi, S)$ has a unique solution $\left(Y_{t}, Z_{t}, K_{t}, V_{t}\right)_{t \leqslant 1}$.

$\mathrm{P}$ r o o f. It will be obtained as the limit of a penalization scheme. Actually for $n \geqslant 0$, let $\left(Y_{t}^{n}, Z_{t}^{n}, V_{t}^{n}\right)_{t \leqslant 1}$ be the $\mathscr{F}_{t}$-adapted process with 
values in $\mathbf{R}^{1+d} \times \mathrm{L}^{2}(\mathbf{U}, d \lambda)$, unique solution of the BSDE associated with $\left(g(t, \omega)+n\left(y-S_{t}\right)^{-}, \xi\right)$, where $x^{-}:=\max (0,-x), x \in \mathbf{R}$, i.e., the triple $\left(Y^{n}, Z^{n}, V^{n}\right)$ satisfies:

$$
\left\{\begin{aligned}
Y^{n} \in & \mathscr{S}^{2}, Z^{n} \in \mathscr{H}^{2, d}, \text { and } V^{n} \in \mathscr{L}^{2}, \\
Y_{t}^{n}= & \xi+\int_{t}^{1} g(s) d s+\int_{t}^{1} n\left(Y_{s}^{n}-S_{s}\right)^{-} d s-\int_{t}^{1} Z_{s}^{n} d B_{s} \\
& -\int_{t}^{1} \int_{\mathbf{U}} V_{s}^{n}(e) \tilde{\mu}(d s, d e), \quad \forall t \leqslant 1 .
\end{aligned}\right.
$$

The existence of such a triple of processes $\left(Y^{n}, Z^{n}, V^{n}\right)$ is due to the Tang-Li result [14]. From now on the proof will be divided into four steps.

Step 1. For any $n \geqslant 0, Y^{n} \leqslant Y^{n+1}$.

For any $t \leqslant 1$, we have

$$
\begin{aligned}
Y_{t}^{n}-Y_{t}^{n+1}= & \int_{t}^{1}\left(Z_{s}^{n}-Z_{s}^{n+1}\right) d B_{s}+\int_{t}^{1} \int_{\mathbf{U}}\left(V_{s}^{n}(e)-V_{s}^{n+1}(e)\right) \tilde{\mu}(d s, d e) \\
& +\int_{t}^{1}\left\{n\left(Y_{s}^{n}-S_{s}\right)^{-}-(n+1)\left(Y_{s}^{n+1}-S_{s}\right)^{-}\right\} d s .
\end{aligned}
$$

But

$$
n\left(Y_{s}^{n}-S_{s}\right)^{-}-(n+1)\left(Y_{s}^{n+1}-S_{s}\right)^{-}=-\left(Y_{s}^{n+1}-S_{s}\right)^{-}+a_{s}^{n}\left(Y_{s}^{n}-Y_{s}^{n+1}\right),
$$

where $\left(a_{s}^{n}\right)_{s \leqslant 1}$ is a $\mathscr{P}$-measurable process such that $\left|a_{s}^{n}\right| \leqslant n$ for all $s \leqslant 1$. Next let us set $\Theta_{t}^{n}=\exp \left\{\int_{0}^{t} a_{s}^{n} d s\right\}$ for $t \leqslant 1$. By Itô's formula we have

$$
d\left(\Theta_{t}^{n}\left(Y_{t}^{n}-Y_{t}^{n+1}\right)\right)=-\left(Y_{t}^{n+1}-S_{t}\right)^{-} \Theta_{t}^{n} d t+d M_{t}^{n}, \quad t \leqslant 1,
$$

where $\left(M_{t}^{n}\right)_{t \leqslant 1}$ is a martingale. Taking the conditional expectation with respect to $\mathscr{F}_{t}$ we obtain $Y^{n} \leqslant Y^{n+1}$, since $Y_{1}^{n+1}-Y_{1}^{n}=0$ and $\Theta^{n}>0$.

Step 2. For any $n \geqslant 0$, the process $Y^{n}$ satisfies

$$
\forall t \leqslant 1, \quad Y_{t}^{n}=\operatorname{essup}_{\tau \geqslant t} \mathbf{E}\left[\int_{t}^{\tau} g(s) d s+\left(Y_{\tau}^{n} \wedge S_{\tau}\right) 1_{[\tau<1]}+\xi 1_{[\tau=1]} \mid \mathscr{F}_{t}\right] .
$$

Indeed for $n \geqslant 0$ and $t \leqslant 1$ we have

$$
\begin{aligned}
Y_{t}^{n}= & \xi+\int_{t}^{1} g(s) d s-\int_{t}^{1} Z_{s}^{n} d B_{s} \\
& +\int_{t}^{1} n\left(Y_{s}^{n}-S_{s}\right)^{-} d s-\int_{t}^{1} \int_{\mathbf{U}} V_{s}^{n}(e) \widetilde{\mu}(d s, d e) .
\end{aligned}
$$

Therefore for any stopping time $\tau \in \mathscr{T}_{t}$ we have

$$
\begin{aligned}
Y_{t}^{n} & =\mathbf{E}\left[Y_{\tau}^{n}+\int_{t}^{\tau} g(s) d s+\int_{t}^{\tau} n\left(Y_{s}^{n}-S_{s}\right)^{-} d s \mid \mathscr{F}_{t}\right] \\
& \geqslant \mathbf{E}\left[\left(S_{\tau} \wedge Y_{\tau}^{n}\right) 1_{[\tau<1]}+\xi 1_{[\tau=1]}+\int_{t}^{\tau} g(s) d s \mid \mathscr{F}_{t}\right],
\end{aligned}
$$


since $Y_{\tau}^{n} \geqslant\left(S_{\tau} \wedge Y_{\tau}^{n}\right) 1_{[\tau<1]}+\xi 1_{[\tau=1]}$. Further for $t \leqslant 1$, let $K_{t}^{n}=\int_{0}^{t} n\left(Y_{s}^{n}-\right.$ $\left.S_{s}\right)^{-} d s$ and let $\tau_{t}^{*}$ be the stopping time defined as follows:

$$
\tau_{t}^{*}=\inf \left\{s \geqslant t: K_{s}^{n}-K_{t}^{n}>0\right\} \wedge 1 .
$$

Then it holds that $1_{\left[\tau_{t}^{*}<1\right]} Y_{\tau_{t}^{*}}^{n}=1_{\left[\tau_{t}^{*}<1\right]} Y_{\tau_{t}^{*}}^{n} \wedge S_{\tau_{t}^{*}}^{n}$. Actually let $\omega$ be fixed such that $\tau_{t}^{*}(\omega)<1$. Therefore there exists a sequence $\left(t_{k}\right)_{k \geqslant 0}$ of real numbers which decreases to $\tau_{t}^{*}(\omega)$ such that $K_{t_{k}}^{n}>K_{t}^{n}$ and $Y_{t_{k}}^{n}(\omega) \leqslant S_{t_{k}}(\omega)$ for all $k \geqslant 0$. Since $Y^{n}$ and $S$ are RCLL processes, taking the limit as $k \rightarrow \infty$ we obtain $Y_{\tau_{t}^{*}}^{n} \leqslant S_{\tau_{t}^{*}}$ which implies $1_{\left[\tau_{t}^{*}<1\right]} Y_{\tau_{t}^{*}}^{n}=1_{\left[\tau_{t}^{*}<1\right]}\left(Y_{\tau_{t}^{*}}^{n} \wedge S_{\tau_{t}^{*}}^{n}\right)$. But from (11) we deduce that

$$
\begin{aligned}
Y_{t}^{n}= & Y_{\tau_{t}^{*}}^{n}+\int_{t}^{\tau_{t}^{*}} g(s) d s-\int_{t}^{\tau_{t}^{*}} Z_{s}^{n} d B_{s}-\int_{t}^{\tau_{t}^{*}} \int_{\mathbf{U}} V_{s}^{n}(e) \widetilde{\mu}(d s, d e) \\
= & 1_{\left[\tau_{t}^{*}<1\right]}\left(Y_{\tau_{t}^{*}}^{n} \wedge S_{\tau_{t}^{*}}\right)+\xi 1_{\left[\tau_{t}^{*}=1\right]}+\int_{t}^{\tau_{t}^{*}} g(s) d s \\
& -\int_{t}^{\tau_{t}^{*}} Z_{s}^{n} d B_{s}-\int_{t}^{\tau_{t}^{*}} \int_{\mathbf{U}} V_{s}^{n}(e) \widetilde{\mu}(d s, d e) .
\end{aligned}
$$

Taking the conditional expectation and using inequality (12) yield for any $n \geqslant 0$ and $t \leqslant 1$,

$$
Y_{t}^{n}=\underset{\tau \in \mathscr{T}_{t}}{\operatorname{esssup}} \mathbf{E}\left[\int_{t}^{\tau} g(s) d s+\left(Y_{\tau}^{n} \wedge S_{\tau}\right) 1_{[\tau<1]}+\xi 1_{[\tau=1]} \mid \mathscr{F}_{t}\right] .
$$

Step 3. There exists an RCLL process $Y=\left(Y_{t}\right)_{t \leqslant 1}$ of $\mathscr{S}^{2}$ such that

$$
Y=\lim _{n \rightarrow \infty} Y^{n} \quad \text { in } \mathscr{H}^{2,1}
$$

and $\mathbf{P}$-a.s., for any $t \leqslant 1$ :

(i) $S_{t} \leqslant Y_{t}$ and $Y_{t}^{n} \nearrow Y_{t}$;

(ii)

$$
Y_{t}=\underset{\tau \in \mathscr{T}_{t}}{\operatorname{ess} \sup } \mathbf{E}\left[\int_{t}^{\tau} g(s) d s+S_{\tau} 1_{[\tau<1]}+\xi 1_{[\tau=1]} \mid \mathscr{F}_{t}\right] \quad\left(Y_{1}=\xi\right) .
$$

Actually for $t \leqslant 1$ let us set

$$
\widetilde{Y}_{t}=\underset{\tau \in \mathscr{T}_{t}}{\operatorname{ess} \sup } \mathbf{E}\left[\int_{t}^{\tau} g(s) d s+S_{\tau} 1_{[\tau<1]}+\xi 1_{[\tau=1]} \mid \mathscr{F}_{t}\right] .
$$

The process $\tilde{Y}$ belongs to $\mathscr{S}^{2}$ since $S$ does, $g \in \mathscr{H}^{2,1}$, and $\xi$ is square integrable. On the other hand, for any $n \geqslant 0$ and $t \leqslant 1$ we obviously have $Y_{t}^{n} \leqslant \widetilde{Y}_{t}$ in consideration of (13). Thus there exits a $\mathscr{P}$-measurable process $Y$ such that $\mathbf{P}$-a.s. for any $t \leqslant 1, Y_{t}^{n} \nearrow Y_{t} \leqslant \tilde{Y}_{t}$ and then $Y=\lim _{n \rightarrow \infty} Y^{n}$ in $\mathscr{H}^{2,1}$. Besides the process $\left(Y_{t}^{n}+\int_{0}^{t} g_{s} d s\right)_{t \leqslant 1}$ is an RCLL supermartingale as 
a Snell envelope of $\left(\int_{0}^{t} g_{s} d s+\left(S_{t} \wedge Y_{t}^{n}\right) 1_{[t<1]}+\xi 1_{[t=1]}\right)_{t \leqslant 1}$ and it converges increasingly to $\left(Y_{t}+\int_{0}^{t} g_{s} d s\right)_{t \leqslant 1}$. It follows that this latter process is also an RCLL supermartingale (see, e.g., [4, p. 86]). Henceforth the process $Y$ is also RCLL and belongs to $\mathscr{S}^{2}$, since it is dominated by $\widetilde{Y}$ which is an element of $\mathscr{S}^{2}$.

Further let us prove that $Y_{t} \geqslant S_{t}$ for any $t \leqslant 1$. Thanks to (10) we have

$$
\mathbf{E}\left[Y_{0}^{n}\right]=\mathbf{E}\left[\xi+\int_{0}^{1} g(s) d s\right]+\mathbf{E}\left[\int_{0}^{1} n\left(Y_{s}^{n}-S_{s}\right)^{-} d s\right] .
$$

Dividing the both sides by $n$ and taking the limit as $n \rightarrow \infty$ we obtain $\mathbf{E}\left[\int_{0}^{1}\left(Y_{s}-S_{s}\right)^{-} d s\right]=0$. The processes $Y$ and $S$ are RCLL, therefore $\mathbf{P}$-a.s., $Y_{t} \geqslant S_{t}$ for $t<1$. Finally since $Y_{1}=\xi \geqslant S_{1}$, we have $Y_{t} \geqslant S_{t}$ for any $t \leqslant 1$.

Let us now show that $Y$ satisfies (14). This a direct consequence of the continuity of the Snell envelope through sequences of increasing RCLL processes (see (A1) in Section 6). Actually on the one hand, the sequence of increasing RCLL processes $\left(\left(S_{t} \wedge Y_{t}^{n}\right) 1_{[t<1]}+\xi 1_{[t=1]}\right)_{t \leqslant 1}$ converges increasingly to the $\operatorname{RCLL}\left(S_{t} 1_{[t<1]}+\xi 1_{[t=1]}\right)_{t \leqslant 1}$, since $Y \geqslant S$. Therefore because of (13) the sequence $\left(\int_{0}^{t} g_{s} d s+Y_{t}^{n}\right)_{t \leqslant 1}$ converges to

$$
\underset{\tau \geqslant t}{\operatorname{ess} \sup } \mathbf{E}\left[\int_{0}^{\tau} g(s) d s+S_{\tau} 1_{[\tau<1]}+\xi 1_{[\tau=1]} \mid \mathscr{F}_{t}\right]
$$

which then is equal to $\left(\int_{0}^{t} g_{s} d s+Y_{t}\right)_{t \leqslant 1}$ and which implies that $Y$ satisfies (14).

Step 4. There exist three processes $Z \in \mathscr{H}^{2, d}, V \in \mathscr{L}^{2}$, and $K \in \mathscr{S}_{i}^{2}$ such that $(Y, Z, V, K)$ is the solution of the BSDE associated with $(g, \xi, S)$.

We know from (14), that the process $\left(\int_{0}^{t} g_{s} d s+Y_{t}\right)_{t \leqslant 1}$ is a Snell envelope which belongs to $\mathscr{S}^{2}$. Then thanks to (A2) (Section 6), there exist a process $K \in \mathscr{S}_{i}^{2}\left(K_{0}=0\right)$ and an $\mathscr{F}_{t}$-martingale $\left(M_{t}\right)_{t \leqslant 1}$ which belongs also to $\mathscr{S}^{2}$ such that

$$
\forall t \leqslant 1, \quad \int_{0}^{t} g_{s} d s+Y_{t}=M_{t}-K_{t} .
$$

Additionally $K=K^{c}+K^{d}$ where $K^{c}$ is continuous nondecreasing and $K^{d}$ is nondecreasing purely discontinuous predictable such that for any $t \leqslant 1$, $\Delta_{t} K^{d}=\left(S_{t-}-Y_{t}\right)^{+} 1_{\left[Y_{t-}=S_{t-}\right]}$.

Since martingale $M$ belongs to $\mathscr{S}^{2}$, the representation property of martingales as stochastic integrals (see, e.g., [12]) implies the existence of two processes $Z$ and $V$ which belong respectively to $\mathscr{H}^{2, d}$ and $\mathscr{L}^{2}$ such that

$$
\text { P-a.s., } \quad \forall t \leqslant 1, \quad M_{t}=M_{0}+\int_{0}^{t}\left\{Z_{s} d B_{s}+\int_{\mathbf{U}} V_{s}(e) \tilde{\mu}(d s, d e)\right\} .
$$

Let us show that $\int_{0}^{1}\left(Y_{s}-S_{s}\right) d K_{s}^{c}=0$. First let us remark that the Snell envelope of $\left(\int_{0}^{t} g_{s} d s+S_{t} 1_{[t<1]}+\xi 1_{[t=1]}+K_{t}^{d}\right)_{t \leqslant 1}$ is nothing else but $\left(\int_{0}^{t} g_{s} d s+\right.$ 
$\left.Y_{t}+K_{t}^{d}\right)_{t \leqslant 1}$. Actually for any $t \leqslant 1$ we have $\int_{0}^{t} g_{s} d s+Y_{t}=M_{t}-K_{t}^{c}-K_{t}^{d}$, therefore the process $\left(\int_{0}^{t} g_{s} d s+Y_{t}+K_{t}^{d}\right)_{t \leqslant 1}$ is also an RCLL supermartingale which dominates the process $\left(\int_{0}^{t} g_{s} d s+S_{t} 1_{[t<1]}+\xi 1_{[t=1]}+K_{t}^{d}\right)_{t \leqslant 1}$. Besides if $\left(N_{t}\right)_{t \leqslant 1}$ is a supermartingale of class [D] which dominates this latter process, then $\left(N_{t}-K_{t}^{d}\right)_{t \leqslant 1}$ is still a supermartingale of class [D] which is greater than $\left(\int_{0}^{t} g_{s} d s+S_{t} 1_{[t<1]}+\xi 1_{[t=1]}\right)_{t \leqslant 1}$. Therefore $\mathbf{P}$-a.s. for any $t \leqslant 1$ we have $N_{t}-$ $K_{t}^{d} \geqslant \int_{0}^{t} g_{s} d s+Y_{t}$ which implies that for all $t \leqslant 1, N_{t} \geqslant \int_{0}^{t} g_{s} d s+Y_{t}+K_{t}^{d}$. It means that the process $\left(\int_{0}^{t} g_{s} d s+Y_{t}+K_{t}^{d}\right)_{t \leqslant T}$ is the smallest supermartingale of class [D] which dominates $\left(\int_{0}^{t} g_{s} d s+S_{t} 1_{[t<1]}+\xi 1_{[t=1]}+K_{t}^{d}\right)_{t \leqslant 1}$ and then it is its Snell envelope (see the characterization given in Section 6).

Further the Snell envelope $\left(\int_{0}^{t} g_{s} d s+Y_{t}+K_{t}^{d}=M_{t}-K_{t}^{c}\right)_{t \leqslant 1}$ of the process $\left(\int_{0}^{t} g_{s} d s+S_{t} 1_{[t<1]}+\xi 1_{[t=1]}+K_{t}^{d}\right)_{t \leqslant 1}$ is regular then for any $t \leqslant 1$, the stopping time $\tau_{t}=\inf \left\{s \geqslant t: K_{s}^{c}>K_{t}^{c}\right\} \wedge 1$ is optimal in the class $\mathscr{T}_{t}$ (see (A3) in Section 6), therefore we have

$$
\int_{t}^{\tau_{t}}\left(Y_{s}+K_{s}^{d}-S_{s}-K_{s}^{d}\right) d K_{s}^{c}=\int_{t}^{\tau_{t}}\left(Y_{s}-S_{s}\right) d K_{s}^{c}=0 .
$$

Since $t$ is arbitrary in $[0,1]$, we have $\int_{0}^{1}\left(Y_{s}-S_{s}\right) d K_{s}^{c}=0$.

Collecting now all those properties yields that the quadruple $(Y, Z, V, K)$ is a solution to the BSDE associated with $(g, \xi, S)$, i.e.,

$$
\left\{\begin{array}{l}
Y \in \mathscr{S}^{2}, Z \in \mathscr{H}^{2, d}, V \in \mathscr{L}^{2}, \text { and } K \in \mathscr{S}_{i}^{2} ; \\
Y_{t}=\xi+\int_{t}^{1} g(s) d s+K_{1}-K_{t}-\int_{t}^{1} Z_{s} d B_{s}-\int_{t}^{1} \int_{\mathbf{U}} V_{s}(e) \widetilde{\mu}(d s, d e) ; \\
\left.Y \geqslant S \text { and if } K^{c} \text { (respectively, } K^{d}\right) \text { is the continuous (respectively, } \\
\quad \text { purely discontinuous) part of } K, \text { then } K^{d} \text { is } \mathscr{P}_{d^{-}} \text {-measurable, } \\
\int_{0}^{1}\left(Y_{t}-S_{t}\right) d K_{t}^{c}=0 \text { and for all } t \leqslant 1, \Delta K_{t}^{d}=\left(S_{t-}-Y_{t}\right)^{+} 1_{\left[Y_{t-}=S_{t-}\right]} .
\end{array}\right.
$$

Theorem 4.1 is proved.

$\mathrm{R}$ e $\mathrm{m}$ a $\mathrm{r} \mathrm{k}$ 4.1. (i) By using the generalization of the monotonic limit theorem of S. Peng [19], one can show that the sequence $\left(Z^{n}\right)_{n \geqslant 0}$ (respectively, $\left.\left(V^{n}\right)_{n \geqslant 0}\right)$ converges weakly in $\mathscr{H}^{2, d}$ (respectively, in $\mathrm{L}^{2}(d t \otimes d \mathbf{P} \otimes d \lambda)$ ) and strongly in $\mathrm{L}^{p}(d t \otimes d \mathbf{P})$ (respectively, in $\mathrm{L}^{2}(d t \otimes d \mathbf{P} \otimes d \lambda)$ ) for any $p<2$. On the other hand, for any stopping time $\tau$ the sequence $\left(K_{\tau}^{n}\right)_{n \geqslant 0}$ converges in $L^{p}(d \mathbf{P})$ to $K_{\tau}$.

(ii) We would have been able to construct the solution of the BSDE (15) directly from (14) in defining the process $Y$, and then $Z, V, K$ as in Step 4 above. This procedure is the well-known Snell envelope method. However we think that proving the convergence of the penalization scheme (10) could be helpful especially when we deal with either numerical schemes for BSDEs of type (15) or viscosity solutions of partial differential-integral equations with discontinuous obstacles. 
4.2. The general case. We are now ready to give the main result of this section. The function $f$ is now general, i.e., it may depend on $(y, z, v)$.

Theorem 4.2. The reflected BSDE with general jumps (3) associated with $(f, \xi, S)$ satisfying assumptions (a)-(c) has a unique solution $(Y, Z, K, V)$.

$\mathrm{P}$ r o o f. It remains to show existence which will be obtained via a fixed point argument. Actually let $\alpha>0$ and $\mathscr{D}:=\mathscr{H}^{2,1} \times \mathscr{H}^{2, d} \times \mathscr{L}^{2}$ endowed with the norm

$$
\|(Y, Z, V)\|_{\alpha}=\left\{\mathbf{E}\left[\int_{0}^{1} e^{\alpha s}\left(\left|Y_{s}\right|^{2}+\left|Z_{s}\right|^{2}+\int_{\mathbf{U}}\left|V_{s}(e)\right|^{2} \lambda(d e)\right) d s\right]\right\}^{1 / 2} .
$$

On the other hand, let $\Phi$ be the mapping from $\mathscr{D}$ into itself which with $(Y, Z, V)$ associates $\Phi(Y, Z, V)=(\underline{Y}, \underline{Z}, \underline{V})$, where $(\underline{Y}, \underline{Z}, \underline{V}, \underline{K})$ is the solution of the reflected BSDE associated with the triple $\left(f\left(t, Y_{t}, Z_{t}, V_{t}\right), \xi, S\right)$. Let $\left(Y^{\prime}, Z^{\prime}, V^{\prime}\right)$ be another triple of $\mathscr{D}$ and $\Phi\left(Y^{\prime}, Z^{\prime}, V^{\prime}\right)=\left(\underline{Y}^{\prime}, \underline{Z}^{\prime}, \underline{V^{\prime}}\right)$. The existence and uniqueness of $(\underline{Y}, \underline{Z}, \underline{V})$ and $\left(\underline{Y}^{\prime}, \underline{Z}^{\prime}, \underline{V}^{\prime}\right)$ are due to Theorem 4.1. By using Itô's formula, we obtain: for any $t \leqslant 1$,

$$
\begin{aligned}
& e^{\alpha t}\left.\underline{Y}_{t}-\underline{Y}_{t}^{\prime}\right)^{2}+\alpha \int_{t}^{1} e^{\alpha s}\left(\underline{Y}_{s}-\underline{Y}_{s}^{\prime}\right)^{2} d s+\int_{t}^{1} e^{\alpha s}\left|\underline{Z}_{s}-\underline{Z}_{s}^{\prime}\right|^{2} d s \\
&+\sum_{t<s \leqslant 1} e^{\alpha s}\left(\Delta_{s}\left(\underline{Y}-\underline{Y}^{\prime}\right)\right)^{2} \\
&=\left(M_{1}-M_{t}\right)+2 \int_{t}^{1} e^{\alpha s}\left(\underline{Y}_{s-}-\underline{Y}_{s-}^{\prime}\right)\left(d \underline{K}_{s}-d \underline{K}_{s}^{\prime}\right) \\
& \quad+2 \int_{t}^{1} e^{\alpha s}\left(\underline{Y}_{s}-\underline{Y}_{s}^{\prime}\right)\left(f\left(s, Y_{s}, Z_{s}, V_{s}\right)-f\left(s, Y_{s}^{\prime}, Z_{s}^{\prime}, V_{s}^{\prime}\right)\right) d s
\end{aligned}
$$

where $\left(M_{t}\right)_{t \leqslant 1}$ is a martingale. But arguments similar to those used in the proof of uniqueness in Proposition 3.1 lead to $\int_{t}^{1} e^{\alpha s}\left(\underline{Y}_{s-}-\underline{Y}_{s-}^{\prime}\right)\left(d \underline{K}_{s}-\right.$ $\left.d \underline{K}_{s}^{\prime}\right) \leqslant 0$, for any $t \leqslant 1$. Therefore taking expectation in (16) and using the inequality $2|a b| \leqslant \varepsilon a^{2}+\varepsilon^{-1} b^{2}$, for any $a, b \in \mathbf{R}$ and $\varepsilon>0$, yield

$$
\begin{aligned}
\alpha \mathbf{E} & {\left[\int_{t}^{1} e^{\alpha s}\left(\underline{Y}_{s}-\underline{Y}_{s}^{\prime}\right)^{2} d s\right]+\mathbf{E}\left[\int_{t}^{1} e^{\alpha s}\left|\underline{Z}_{s}-\underline{Z}_{s}^{\prime}\right|^{2} d s\right] } \\
& +\mathbf{E}\left[\int_{t}^{1} e^{\alpha s} d s \int_{\mathbf{U}}\left(\underline{V}_{s}(e)-\underline{V}_{s}^{\prime}(e)\right)^{2} \lambda(d e)\right] \\
\leqslant & 2 \mathbf{E}\left[\int_{t}^{1} e^{\alpha s}\left(\underline{Y}_{s}-\underline{Y}_{s}^{\prime}\right)\left(f\left(s, Y_{s}, Z_{s}, V_{s}\right)-f\left(s, Y_{s}^{\prime}, Z_{s}^{\prime}, V_{s}^{\prime}\right)\right) d s\right] \\
\leqslant & \frac{C_{f}}{\varepsilon} \mathbf{E}\left[\int_{t}^{1} e^{\alpha s}\left\{\left(Y_{s}-Y_{s}^{\prime}\right)^{2}+\left|Z_{s}-Z_{s}^{\prime}\right|^{2}+\int_{\mathbf{U}}\left|V_{s}(e)-V_{s}^{\prime}(e)\right|^{2} \lambda(d e)\right\} d s\right] \\
& +3 \varepsilon C_{f} \mathbf{E}\left[\int_{t}^{1} e^{\alpha s}\left(\underline{Y}_{s}-\underline{Y}_{s}^{\prime}\right)^{2} d s\right]
\end{aligned}
$$


and then

$$
\begin{aligned}
(\alpha- & \left.3 \varepsilon C_{f}\right) \mathbf{E}\left[\int_{t}^{1} e^{\alpha s}\left(\underline{Y}_{s}-\underline{Y}_{s}^{\prime}\right)^{2} d s\right] \\
& +\mathbf{E}\left[\int_{t}^{1} e^{\alpha s}\left|\underline{Z}_{s}-\underline{Z}_{s}^{\prime}\right|^{2} d s+\int_{t}^{1} e^{\alpha s} d s \int_{\mathbf{U}}\left(\underline{V}_{s}(e)-\underline{V}_{s}^{\prime}(e)\right)^{2} \lambda(d e)\right] \\
\leqslant & \frac{C_{f}}{\varepsilon} \mathbf{E}\left[\int_{t}^{1} e^{\alpha s}\left\{\left|Y_{s}-Y_{s}^{\prime}\right|^{2}+\left|Z_{s}-Z_{s}^{\prime}\right|^{2}+\int_{\mathbf{U}}\left|V_{s}(e)-V_{s}^{\prime}(e)\right|^{2} \lambda(d e)\right\} d s\right] .
\end{aligned}
$$

Next by taking $\varepsilon=2 C_{f}$ and $\alpha=1+6 C_{f}^{2}$, we get that $\Phi$ is a contraction on $\left(\mathscr{D},\|\cdot\|_{1+6 C_{f}^{2}}\right)$. Therefore it has a fixed point $(Y, Z, V)$, i.e., $\Phi(Y, Z, V)=$ $(Y, Z, V)$ and then, with the associated process $K$, the quadruple $(Y, Z, V, K)$ is the unique solution of the reflected BSDE associated with $(f, \xi, S)$ since $Y \in \mathscr{S}^{2}$. Theorem 4.2 is proved.

4.3. On the regularity of the process $K$. We next focus on the regularity of the process $K$ of the solution of the BSDE (3).

Proposition 4.1. Let $(Y, Z, K, V)$ be the unique solution of the reflected BSDE (15) associated with $(g(t), \xi, S)$, and consider the following assertions:

(i) $K$ is continuous $\left(K^{d} \equiv 0\right)$;

(ii) the sequence of processes $\left(Y^{n}\right)_{n \geqslant 0}$ of (10) converges uniformly to $Y$;

(iii) $\mathbf{E}\left[\int_{0}^{1}\left|Z_{s}^{n}-Z_{s}\right|^{2} d s+\int_{0}^{1} \int_{\mathbf{U}}\left|V_{s}^{n}(e)-V_{s}(e)\right|^{2} \lambda(d e) d s\right] \rightarrow 0$.

Then (i) and (ii) are equivalent and statement (ii) implies (iii).

P r o o f. $(\mathrm{i}) \Rightarrow(\mathrm{ii})$. Assume that (i) is fulfilled. Therefore the jumps of $Y$ are the same as the ones of its stochastic integral with respect to $\widetilde{\mu}$. It follows that for any $t \leqslant 1,{ }^{p} Y_{t}=Y_{t-}$, where ${ }^{p} Y$ is the predictable projection of $Y$, since in that case $Y$ has only inaccessible jumps. The same happens with $Y^{n}$. As $Y^{n} \nearrow Y$, then ${ }^{p} Y^{n} \nearrow{ }^{p} Y$, that is to say, $Y_{t-}^{n} \nearrow Y_{t-}$, for all $t \leqslant 1$. It follows from a generalized Dini's Theorem (see [4, p. 203]), that $\mathbf{P}$-a.s. the sequence $\left(Y^{n}\right)_{n \geqslant 0}$ converges uniformly to $Y$.

(ii) $\Rightarrow(\mathrm{i})$. If $\left(Y^{n}\right)_{n \geqslant 0}$ converges uniformly to $Y$, then $Y_{-}^{n} \nearrow Y_{-}$and ${ }^{p} Y^{n} \nearrow^{p} Y$. But $Y^{n}$ is RCLL and has only inaccessible jumps, then ${ }^{p} Y^{n}=Y_{-}^{n}$ which implies that ${ }^{p} Y=Y_{-}$. Therefore the Snell envelope $\left(\int_{0}^{t} f\left(s, Y_{s}, Z_{s}, V_{s}\right) d s+Y_{t}\right)_{t \leqslant 1}$ is regular and then $K^{d}=0$ (see (A3) in Section 6) which is the claim.

(ii) $\Rightarrow$ (iii). If the sequence $\left(Y^{n}\right)_{n \geqslant 0}$ converges uniformly to $Y$, then thanks to the dominated convergence theorem we have also $\mathbf{E}\left[\sup _{t \leqslant 1}\left(Y_{t}-\right.\right.$ $\left.\left.Y_{t}^{n}\right)^{2}\right] \searrow 0$. In order to get the result, it is enough to apply Itô's formula with $\left(Y-Y^{n}\right)^{2}$ after having noticed that $\mathbf{E}\left[\sup _{t \leqslant 1}\left(\left(S_{t}-Y_{t}^{n}\right)^{+}\right)^{2}\right] \rightarrow 0$, since $Y \geqslant S$. Therefore we have

$$
\mathbf{E}\left[\int_{0}^{1}\left|Z_{s}^{n}-Z_{s}\right|^{2} d s+\int_{0}^{1} \int_{\mathbf{U}}\left|V_{s}^{n}(e)-V_{s}(e)\right|^{2} \lambda(d e) d s\right] \rightarrow 0 .
$$


$\mathrm{R}$ e $\mathrm{m}$ a $\mathrm{r} \mathrm{k}$ 4.3. Let us point out that the implication (iii) $\Rightarrow$ (i) is not true in general. Actually let us consider the following deterministic counterexample. Assume $\xi=1 / 2, f \equiv 0$, and $S_{t}=1_{[t<1 / 2]}$. Therefore the solution of the BSDE associated with $(f, \xi, S)$ is $Z \equiv 0, V \equiv 0, Y_{t}=1_{[t<1 / 2]}+$ $(1 / 2) 1_{[t \geqslant 1 / 2]}$ and $K_{t}=K_{t}^{d}=(1 / 2) 1_{[1 / 2 \leqslant t \leqslant 1]}$. Indeed it is easily seen that for any $t \leqslant 1$, we have $Y_{t}=1 / 2+K_{1}^{d}-K_{t}^{d}$. So obviously the statement (iii) holds true, since $Y_{t}^{n}=\left(1-e^{n(t-1 / 2)} / 2\right) 1_{[t<1 / 2]}+(1 / 2) 1_{[t \geqslant 1 / 2]}, t \leqslant 1, Z^{n}=Z=0$, $V^{n}=V=0$ but $K$ (and also $Y$ ) is not continuous.

5. BSDEs with two discontinuous reflecting barriers. We now consider the problem of reflection with respect to two barriers, an upper and a lower ones. For that let us consider two processes $L:=\left(L_{t}\right)_{t \leqslant 1}$ and $U:=\left(U_{t}\right)_{t \leqslant 1}$ which stand for the barriers where «the solution» is reflected in between and which satisfy:

(i) $L$ and $U$ belong to $\mathscr{S}^{2}$ and $\mathbf{P}$-a.s., for any $t \leqslant 1, L_{t} \leqslant U_{t}$;

(ii) there exist two nonnegative supermartingales $\left(h_{t}\right)_{t \leqslant 1}$ and $\left(h_{t}^{\prime}\right)_{t \leqslant 1}$ of $\mathscr{S}^{2}$ such that

$$
\forall t \leqslant 1, \quad L_{t} \leqslant h_{t}-h_{t}^{\prime} \leqslant U_{t}
$$

(iii) for any $t<1, L_{t-}<U_{t-}$ and $L_{t}<U_{t}$.

Assumption (ii) is the well-known Mokobodski hypothesis. Hereafter we assume those assumptions hold.

Let us now introduce the BSDE associated with $(f, \xi, L, U)$. A solution is a quintuple of processes $\left(Y_{t}, Z_{t}, K_{t}^{+}, K_{t}^{-}, V_{t}\right)_{t \leqslant 1}$ which satisfies:

$$
\begin{aligned}
& \text { (a) } Y \in \mathscr{S}^{2}, K^{ \pm} \in \mathscr{S}_{i}^{2}, Z \in \mathscr{H}^{2, d} \text {, and } V \in \mathscr{L}^{2} \text {; } \\
& \text { (b) }-d Y_{t}=f\left(t, Y_{t}, Z_{t}, V_{t}\right) d t+d K_{t}^{+}-d K_{t}^{-} \\
& -Z_{t} d B_{t}-\int_{\mathbf{U}} V_{t}(e) \tilde{\mu}(d t, d e), t \leqslant 1 \text {, and } Y_{1}=\xi ;
\end{aligned}
$$

Note that BSDE (17) may not have a solution for general processes $L, U$. Actually if, for example, $L$ is not a semimartingale, and $L, U$ coincide, then obviously the equation cannot have a solution, since the process $Y$ is always a semimartingale.

First we are going to focus on the issue of uniqueness of the solution of (17).

Proposition 5.1. The BSDE with two reflecting barriers (17) associated with $(f, \xi, L, U)$ has at most one solution. 
P r o o f. Assume that $\left(Y, Z, K^{ \pm}, V\right)$ and $\left(Y^{\prime}, Z^{\prime}, K^{\prime \pm}, V^{\prime}\right)$ are two solutions of (17). First let us show that for any $t \leqslant 1$,

$$
\int_{t}^{1}\left(Y_{s-}-Y_{s-}^{\prime}\right)\left(d K_{s}-d K_{s}^{\prime}\right) \leqslant 0,
$$

where $K=K^{+}-K^{-}$and $K^{\prime}=K^{{ }^{+}}-K^{\prime-}$. For any $t \leqslant 1$ we have

$$
\begin{aligned}
& \int_{] t, 1]}\left(Y_{s-}-Y_{s-}^{\prime}\right)\left(d K_{s}-d K_{s}^{\prime}\right)=\int_{] t, 1]}\left(Y_{s-}-Y_{s-}^{\prime}\right)\left(d K_{s}^{c}-d K_{s}^{\prime c}\right) \\
& +\int_{] t, 1]}\left(Y_{s-}-Y_{s-}^{\prime}\right)\left(d K_{s}^{d}-d K_{s}^{\prime d}\right) .
\end{aligned}
$$

The processes $Y$ and $Y^{\prime}$ belong to $\mathscr{S}^{2}$ and their jumps $\delta(\omega):=\{t \in$ $\left.[0,1], \Delta_{t} Y(\omega) \neq 0\right\}$ and $\delta^{\prime}(\omega):=\left\{t \in[0,1], \Delta_{t} Y^{\prime}(\omega) \neq 0\right\}$ are countable at most. Therefore

$$
\begin{aligned}
\int_{] t, 1]}\left(Y_{s-}-Y_{s-}^{\prime}\right)\left(d K_{s}^{c}-d K_{s}^{\prime c}\right)= & \int_{] t, 1]}\left(Y_{s}-Y_{s}^{\prime}\right)\left(d K_{s}^{+c}-d K_{s}^{\prime+c}\right) \\
& -\int_{] t, 1]}\left(Y_{s}-Y_{s}^{\prime}\right)\left(d K_{s}^{-c}-d K_{s}^{\prime-c}\right) .
\end{aligned}
$$

But since for any $t \leqslant 1,\left(Y_{t}-L_{t}\right) d K_{t}^{+c}=0$, then

$$
\begin{aligned}
\int_{] t, 1]} & \left(Y_{s}-Y_{s}^{\prime}\right)\left(d K_{s}^{+c}-d K_{s}^{\prime+c}\right) \\
& =-\int_{] t, 1]}\left(Y_{s}-L_{s}\right) d K_{s}^{\prime+c}+\int_{] t, 1]}\left(L_{s}-Y_{s}^{\prime}\right) d K_{s}^{+c} \leqslant 0 .
\end{aligned}
$$

In the same way, we have also $\int_{] t, 1]}\left(Y_{s}-Y_{s}^{\prime}\right)\left(d K_{s}^{-c}-d K_{s}^{\prime-c}\right) \geqslant 0$. Therefore for any $t \leqslant 1$,

$$
\int_{] t, 1]}\left(Y_{s-}-Y_{s-}^{\prime}\right)\left(d K_{s}^{c}-d K_{s}^{c}\right) \leqslant 0 .
$$

Let us now focus on the discontinuous parts of $K-K^{\prime}$. For all $t \leqslant 1$,

$$
\begin{aligned}
\int_{] t, 1]}\left(Y_{s-}-Y_{s-}^{\prime}\right)\left(d K_{s}^{d}-d K_{s}^{\prime d}\right)= & \int_{] t, 1]}\left(Y_{s-}-Y_{s-}^{\prime}\right)\left(d K_{s}^{+d}-d K_{s}^{\prime+d}\right) \\
& -\int_{] t, 1]}\left(Y_{s-}-Y_{s-}^{\prime}\right)\left(d K_{s}^{-d}-d K_{s}^{\prime-d}\right) \leqslant 0 .
\end{aligned}
$$

Indeed

$$
\begin{aligned}
\int_{] t, 1]} & \left(Y_{s-}-Y_{s-}^{\prime}\right)\left(d K_{s}^{+d}-d K_{s}^{\prime+d}\right) \\
& =\int_{] t, 1]}\left(L_{s-}-Y_{s-}^{\prime}\right) d K_{s}^{+d}-\int_{] t, 1]}\left(Y_{s-}-L_{s-}\right) d K_{s}^{\prime+d} \leqslant 0
\end{aligned}
$$


since $Y \geqslant L$ (respectively, $Y^{\prime} \geqslant L$ ) and the jumps of $K^{+d}$ (respectively, $K^{\prime+d}$ ) occur only when $Y_{-}=L_{-}$(respectively, $Y_{-}^{\prime}=L_{-}$). In the same way we have $\int_{\mid t, 1]}\left(Y_{s-}-Y_{s-}^{\prime}\right)\left(d K_{s}^{-d}-d K_{s}^{\prime-d}\right) \geqslant 0$, and then we get $(21)$. Combining now (19), (20), and (21) we obtain (18).

Now by using Itô's formula with $\left(Y-Y^{\prime}\right)^{2}$ and following the same steps as in the proof of Proposition 3.1, we obtain that $Y=Y^{\prime}, Z=Z^{\prime}$, $V=V^{\prime}$ and finally $K=K^{\prime}$. Thus, thanks to their expressions, we have also $K^{+d}=K^{\prime+d}$ and $K^{-d}=K^{\prime-d}$ and then $K^{+c}-K^{-c}=K^{+c}-K^{\prime-c}$. It remains to show that $K^{+c}=K^{\prime+c}$ and $K^{-c}=K^{\prime-c}$. But for any $t \leqslant 1$ we have

$$
\begin{aligned}
\int_{0}^{t}\left(Y_{s}-L_{s}\right) d\left(K_{s}^{+c}-K_{s}^{-c}\right) & =-\int_{0}^{t}\left(U_{s}-L_{s}\right) d K_{s}^{-c} \\
& =\int_{0}^{t}\left(Y_{s}^{\prime}-L_{s}\right) d\left(K_{s}^{\prime+c}-K_{s}^{\prime-c}\right) \\
& =-\int_{0}^{t}\left(U_{s}-L_{s}\right) d K_{s}^{\prime-c}
\end{aligned}
$$

Therefore $K^{-c}=K^{\prime-c}$, since for any $t<1, L_{t}<U_{t}$ and then $K^{+c}=K^{\prime+c}$. Thus the solution of (17) is unique. Proposition 5.1 is proved.

Once again to show that equation (17) has a solution we are going first to suppose that $f$ does not depend on $(y, z, v)$, i.e., $f(t, \omega, y, z, v)=f(t)$. We have the following theorem.

Theorem 5.1. There exist processes $\left(Y_{t}, Z_{t}, K_{t}^{+}, K_{t}^{-}, V_{t}\right)_{t \leqslant 1}$ solution of the BSDE with two reflecting barriers associated with $(f(t), \xi, L, U)$.

$\mathrm{P}$ r o o f. Even if the barriers have predictable jumps, the proof of this theorem, in its main steps, is classical (see, e.g., [3], [10]). Consider the following processes defined by: $\forall t \leqslant 1$,

$$
\begin{aligned}
& H_{t}=\left(h_{t}+\mathbf{E}\left[\xi^{-} \mid F_{t}\right]\right) 1_{[t<1]}+\mathbf{E}\left[\int_{t}^{1} f(s)^{-} d s \mid \mathscr{F}_{t}\right], \\
& \Theta_{t}=\left(h_{t}^{\prime}+\mathbf{E}\left[\xi^{+} \mid F_{t}\right]\right) 1_{[t<1]}+\mathbf{E}\left[\int_{t}^{1} f(s)^{+} d s \mid \mathscr{F}_{t}\right] \\
& \widetilde{L}_{t}=L_{t} 1_{[t<1]}+\xi 1_{[t=1]}-\mathbf{E}\left[\xi+\int_{t}^{1} f(s) d s \mid \mathscr{F}_{t}\right] \\
& \widetilde{U}_{t}=U_{t} 1_{[t<1]}+\xi 1_{[t=1]}-\mathbf{E}\left[\xi+\int_{t}^{1} f(s) d s \mid \mathscr{F}_{t}\right]
\end{aligned}
$$

where $f(t)^{-}=\max \{-f(t), 0\}$ and $f(t)^{+}=\max \{f(t), 0\}$. Since $h$ and $h^{\prime}$ are nonnegative supermartingales, the processes $H$ and $\Theta$ are also nonnegative supermartingales which moreover belong to $\mathscr{S}^{2}$ and satisfy $H_{1}=\Theta_{1}=0$. On the other hand, thanks to Mokobodski's hypothesis, we can easily verify that for any $t \leqslant 1$

$$
\widetilde{L} \leqslant H-\Theta \leqslant \widetilde{U}
$$


Next let us consider the sequences $\left(N_{n}^{ \pm}\right)_{n \geqslant 0}$ of processes defined recursively as follows: $N_{0}^{ \pm}=0$ and for $n \geqslant 0$,

$$
N^{+, n+1}=R\left(N^{-, n}+\widetilde{L}\right) \quad \text { and } \quad N^{-, n+1}=R\left(N^{+, n}-\widetilde{U}\right),
$$

where $R$ is the Snell envelope operator (see Section 6). Now by induction and using (22) we can easily verify that for any $n \geqslant 0$,

$$
0 \leqslant N^{+, n} \leqslant N^{+, n+1} \leqslant H \quad \text { and } \quad 0 \leqslant N^{-, n} \leqslant N^{-, n+1} \leqslant \Theta .
$$

It follows that the sequence $\left(N_{n}^{+}\right)_{n \geqslant 0}$ (respectively, $\left(N_{n}^{-}\right)_{n \geqslant 0}$ ) converges pointwise to a supermartingale $N^{+}$(respectively, $N^{-}$) (see, e.g., [4, p. 86]). In addition $N^{+}$and $N^{-}$belong to $\mathscr{S}^{2}$ and verify (for more details see (A1) in Section 6)

$$
N^{+}=R\left(N^{-}+\widetilde{L}\right) \quad \text { and } \quad N^{-}=R\left(N^{+}-\widetilde{U}\right) .
$$

Further the Doob-Meyer decomposition of $N^{ \pm}$yields:

$$
\forall t \leqslant 1, \quad N_{t}^{ \pm}=M_{t}^{ \pm}-K_{t}^{ \pm}
$$

where $M^{ \pm}$are RCLL martingales and $K^{ \pm}$nondecreasing processes such that $K_{0}^{ \pm}=0$. Moreover since $N^{ \pm} \in \mathscr{S}^{2}$, we have $\mathbf{E}\left[\left(K_{1}^{ \pm}\right)^{2}\right]<\infty$ (see (A2) in Section 6). Therefore $M^{ \pm}$belong also to $\mathscr{S}^{2}$ and then there exist processes $Z^{ \pm} \in \mathscr{H}^{2, d}$ and $V^{ \pm} \in \mathscr{L}^{2}$ such that (see [12])

$$
\forall t \leqslant 1, \quad M_{t}^{ \pm}=M_{0}^{ \pm}+\int_{0}^{t}\left\{Z_{s}^{ \pm} d B_{s}+\int_{\mathbf{U}} V_{s}^{ \pm}(e) \widetilde{\mu}(d s, d e)\right\} .
$$

Let us denote by $K^{ \pm d}$ (respectively, $K^{ \pm c}$ ) the purely discontinuous (respectively, continuous) part of $K^{ \pm}$. Similarly to the case of BSDEs with one reflecting barrier (see Section 4, Step 4) we have:

$$
\int_{0}^{1}\left(N_{s}^{+}-N_{S}^{-}-\widetilde{L}_{s}\right) d K_{s}^{+c}=\int_{0}^{1}\left(N_{s}^{-}-N_{s}^{+}+\widetilde{L}_{s}\right) d K_{s}^{-c}=0
$$

On the other hand, the processes $K^{ \pm d}$ are predictable and if $\tau$ is a predictable stopping time (see (A2) in Section 6), then

$$
\begin{aligned}
& \left\{\Delta K_{\tau}^{+d}>0\right\} \subset\left\{N_{\tau-}^{+}=N_{\tau-}^{-}+\widetilde{L}_{\tau-}\right\} \\
& \left\{\Delta K_{\tau}^{-d}>0\right\} \subset\left\{N_{\tau-}^{-}=N_{\tau-}^{+}-\widetilde{U}_{\tau-}\right\}
\end{aligned}
$$

But $L_{-}<U_{-}$and $\tau$ is predictable then we have $\widetilde{L}_{\tau-}<\widetilde{U}_{\tau-}$ since the jumps of martingales with respect to $(\mathscr{F})_{t \leqslant 1}$ are inaccessible because they come only from the Poisson random measure $\mu$. Therefore the predictable 
processes $K^{+d}$ and $K^{-d}$ cannot jump in the same time otherwise we would have $\widetilde{L}_{\tau-}=\widetilde{U}_{\tau-}$ which is impossible due to Assumption (iii). Henceforth

$$
\begin{aligned}
& \left\{\Delta K_{\tau}^{+d}>0\right\} \subset\left\{N_{\tau-}^{+}=N_{\tau}^{-}+\widetilde{L}_{\tau-}\right\}, \\
& \left\{\Delta K_{\tau}^{-d}>0\right\} \subset\left\{N_{\tau-}^{-}=N_{\tau}^{+}-\widetilde{U}_{\tau-}\right\} .
\end{aligned}
$$

It follows that for any $t \leqslant 1$ we have

$$
\Delta K_{t}^{+d}=\left(N_{t-}^{+}-N_{t}^{+}\right)^{+} 1_{\left[N_{t-}^{+}=N_{t-}^{-}+\widetilde{L}_{t-}\right]}=\left(N_{t}^{-}+\widetilde{L}_{t-}-N_{t}^{+}\right)^{+} 1_{\left[N_{t-}^{+}=N_{t-}^{-}+\widetilde{L}_{t-}\right]} .
$$

In the same way we obtain for any $t \leqslant 1$,

$$
\Delta K_{t}^{-d}=\left(N_{t-}^{-}-N_{t}^{-}\right)^{+} 1_{\left[N_{t-}^{-}=N_{t-}^{+}-\widetilde{U}_{t-}\right]}=\left(N_{t}^{+}-\widetilde{U}_{t-}-N_{t}^{-}\right)^{+} 1_{\left[N_{t-}^{-}=N_{t-}^{+}-\widetilde{U}_{t-}\right]} .
$$

Finally for $t \leqslant 1$, let us set

$$
\begin{aligned}
& Y_{t}=N_{t}^{+}-N_{t}^{-}+\mathbf{E}\left[\xi+\int_{t}^{1} f(s) d s \mid \mathscr{F}_{t}\right], \\
& Z_{t}=Z_{t}^{+}-Z_{t}^{-}+\eta_{t}, \quad V_{t}=V_{t}^{+}-V_{t}^{-}+\rho_{t},
\end{aligned}
$$

where the processes $\eta$ and $\rho$ are such that for all $t \leqslant 1$,

$$
\begin{aligned}
\mathbf{E}\left[\xi+\int_{0}^{1} f(s) d s \mid \mathscr{F}_{t}\right]= & \mathbf{E}\left[\xi+\int_{0}^{1} f(s) d s\right] \\
& +\int_{0}^{t}\left\{\eta_{s} d B_{s}+\int_{\mathbf{U}} \rho_{s}(e) \widetilde{\mu}(d s, d e)\right\} .
\end{aligned}
$$

Therefore the quintuple $\left(Y, Z, V, K^{+}, K^{-}\right)$is the solution of the BSDE with two reflecting barriers associated with $(f(t), \xi, L, U)$, i.e.,

(i) $Y \in \mathscr{S}^{2}, K^{ \pm} \in \mathscr{S}_{i}^{2}, Z \in \mathscr{H}^{2, d}$, and $V \in \mathscr{L}^{2}$;

(ii) $-d Y_{t}=f(t) d t+d K_{t}^{+}-d K_{t}^{-}$

$$
-Z_{t} d B_{t}-\int_{\mathbf{U}} V_{t}(e) \tilde{\mu}(d t, d e), t \leqslant 1, \text { and } Y_{1}=\xi ;
$$

(iii) for all $t \leqslant 1, L_{t} \leqslant Y_{t} \leqslant U_{t}$ and if $K^{ \pm c}$ is the continuous part of $K^{ \pm}$, then $\left(Y_{t}-L_{t}\right) d K_{t}^{+c}=0$ and $\left(U_{t}-Y_{t}\right) d K_{t}^{-c}=0$;

(iv) if $K^{ \pm d}$ denotes the purely discontinuous part of $K^{ \pm}$, then $K^{ \pm d}$ is $\mathscr{P}_{d^{-}}$measurable and, for all $t \leqslant 1, \Delta K_{t}^{+d}=\left(L_{t-}-Y_{t}\right)^{+} 1_{\left[Y_{t-}=L_{t-}\right]}$ and $\Delta K_{t}^{-d}=\left(Y_{t}-U_{t-}\right)^{+} 1_{\left[Y_{t-}=U_{t-}\right]}$.

Theorem 5.1 is proved.

We are now ready to give the main result of this section.

Theorem 5.2. The reflected BSDE (17) associated with $(f(t, y, z, v)$, $\xi, L, U)$ has a unique solution $\left(Y, Z, V, K^{+}, K^{-}\right)$. 
P r o o f. We give a brief proof, since once more it is somehow classical. Let $\mathscr{H}:=\mathscr{H}^{2,1} \times \mathscr{H}^{2, d} \times \mathscr{L}^{2}$, and let $\Phi$ be the following application:

$$
\begin{aligned}
\Phi: & \mathscr{H} \rightarrow \mathscr{H} \\
& (y, z, v) \mapsto \Phi(y, z, v)=(\bar{Y}, \bar{Z}, \bar{V}),
\end{aligned}
$$

where $(\bar{Y}, \bar{Z}, \bar{V})$ is the triple for which there exist two other processes $\bar{K}^{ \pm}$ which belong to $\mathscr{S}^{2}$ such that $\left(\bar{Y}, \bar{Z}, \bar{V}, \bar{K}^{+}, \bar{K}^{-}\right)$is a solution for the BSDE with two reflecting barriers associated with $\left(f\left(t, y_{t}, z_{t}, v_{t}\right), \xi, L, U\right)$. Now let $\alpha>0,\left(y^{\prime}, v^{\prime}, z^{\prime}\right) \in \mathscr{H}$, and $\left(\bar{Y}^{\prime}, \bar{Z}^{\prime}, \bar{V}^{\prime}\right)=\Phi\left(y^{\prime}, z^{\prime}, v^{\prime}\right)$. Using Itô's formula and taking into account that $e^{\alpha s}\left(\bar{Y}_{s}-\bar{Y}_{s}^{\prime}\right) d\left(\bar{K}_{s}^{+}-\bar{K}_{s}^{-}-\bar{K}_{s}^{\prime+}+\bar{K}_{s}^{\prime-}\right) \leqslant 0$, for any $s \leqslant 1$, we can prove the existence of a constant $\bar{C}<1$, by an appropriate choice of $\alpha$ (see, e.g., [10], [11]), such that:

$$
\begin{gathered}
\mathbf{E}\left[\int_{0}^{1} e^{\alpha s}\left\{\left(\bar{Y}_{s}-\bar{Y}_{s}^{\prime}\right)^{2}+\left|\bar{Z}_{s}-\bar{Z}_{s}^{\prime}\right|^{2}+\int_{\mathbf{U}}\left|\bar{V}_{s}(e)-\bar{V}_{s}^{\prime}(e)\right|^{2} \lambda(d e)\right\} d s\right] \\
\leqslant \bar{C} \mathbf{E}\left[\int_{0}^{1} e^{\alpha s}\left\{\left|y_{s}-y_{s}^{\prime}\right|^{2}+\left|z_{s}-z_{s}^{\prime}\right|^{2}+\left\|v_{s}-v_{s}^{\prime}\right\|^{2}\right\} d s\right] .
\end{gathered}
$$

Thus the mapping $\Phi$ is a contraction and then has a unique fixed point $(Y, Z, V)$ which actually belongs to $\mathscr{S}^{2} \times \mathscr{H}^{2, d} \times \mathscr{L}^{2}$. Moreover there exist $K^{ \pm} \in \mathscr{S}^{2}\left(K_{0}^{ \pm}=0\right)$ such that $\left(Y, Z, V, K^{+}, K^{-}\right)$is the unique solution of the reflected BSDE associated with $(f, \xi, L, U)$. Theorem 5.2 is proved.

6. Appendix. In this appendix, all notation are the same as in the previous sections.

Let $\eta:=\left(\eta_{t}\right)_{t \leqslant 1}$ be an RCLL, $\mathscr{P}$-measurable process with values in $\mathbf{R}$ and of a class $[\mathrm{D}]$. The Snell envelope of the process $\eta$, which we denote by $R(\eta):=\left(R(\eta)_{t}\right)_{t \leqslant 1}$ is the smallest RCLL $\mathscr{F}_{t}$-supermartingale of class [D] which dominates $\eta$, i.e., $\mathbf{P}$-a.s., for any $t \leqslant 1, R(\eta)_{t} \geqslant \eta_{t}$. It has the following expression (see, e.g., [5] for more details):

$$
\text { P-a.s., } \quad \forall t \leqslant 1, \quad R(\eta)_{t}=\underset{\tau \geqslant t}{\operatorname{ess} \sup } \mathbf{E}\left[\eta_{\tau} \mid \mathscr{F}_{t}\right] \quad\left(R(\eta)_{1}=\eta_{1}\right) .
$$

We now give some properties of the Snell envelope of processes.

(A1) Let $\left(U^{n}\right)_{n \geqslant 0}$ be a nondecreasing sequence of $\mathscr{P}$-measurable, $R C L L$, $\mathbf{R}$-valued processes of class $[\mathrm{D}]$ which converges pointwise to $U$ another $R C L L, \mathbf{R}$-valued, $\mathscr{P}$-measurable process of class $[\mathrm{D}]$. Then $\mathbf{P}$-a.s., for any $t \leqslant 1, R\left(U^{n}\right)_{t} \nearrow R(U)_{t}$.

P r o o f. Actually for any $n \geqslant 0, \mathbf{P}$-a.s. for any $t \leqslant 1, R\left(U^{n}\right)_{t} \leqslant$ $R(U)_{t}$. Therefore $\mathbf{P}$-a.s., for any $t \leqslant 1$, $\lim _{n \rightarrow \infty} R\left(U^{n}\right)_{t} \leqslant R(U)_{t}$. Note that the process $\left(\lim _{n \rightarrow \infty} R\left(U^{n}\right)_{t}\right)_{t \leqslant 1}$ is an RCLL supermartingale of class $[D]$, since it is a limit of a nondecreasing sequence of supermartingales (see, e.g., [4, p. 86]). But $U^{n} \leqslant R\left(U^{n}\right)$ implies that $\mathbf{P}$-a.s., for any $t \leqslant 1, U_{t} \leqslant$ 
$\lim _{n \rightarrow \infty} R\left(U^{n}\right)_{t}$ and then $R(U)_{t} \leqslant \lim _{n \rightarrow \infty} R\left(U^{n}\right)_{t}$, since the Snell envelope of $U$ is the lowest supermartingale which dominates $U$. It follows that $\mathbf{P}$-a.s., for any $t \leqslant 1, \lim _{n \rightarrow \infty} R\left(U^{n}\right)_{t}=R(U)_{t}$, whence the desired result.

(A2) (Doob-Meyer decomposition of a Snell envelope). Let $\eta:=\left(\eta_{t}\right)_{t \leqslant 1}$ be an RCLL, P्P-measurable process with values in $\mathbf{R}$ and of a class [D], and $R(\eta):=\left(R(\eta)_{t}\right)_{t \leqslant 1}$ its Snell envelope. Then there exist an $R C L L$ $\mathscr{F}_{t}$-martingale $\left(M_{t}\right)_{t \leqslant 1}$ and a nondecreasing $R C L L \mathscr{F}_{t}$-predictable process $\left(K_{t}\right)_{t \leqslant 1}\left(K_{0}=0\right)$ such that

$$
\text { P-a.s., } \quad \forall t \leqslant 1, \quad R(\eta)_{t}=M_{t}-K_{t} .
$$

Moreover we have:

(i) if $R(\eta)$ belongs also to $\mathscr{S}^{2}$, then $\mathbf{E}\left[K_{1}^{2}\right]<\infty$;

(ii) if $K^{c}$ (respectively, $K^{d}$ ) denotes the continuous (respectively, purely discontinuous) part of $K$, then $K^{d}$ is $\mathscr{F}_{t}$-predictable, $\left\{\Delta K^{d}>0\right\} \subset$ $\left\{R(\eta)_{-}=\eta_{-}\right\}$, and $\Delta_{t} K^{d}=\left(\eta_{t-}-R(\eta)_{t}\right)^{+} 1_{\left[R(\eta)_{t-}=\eta_{t-}\right]}$.

P r o o f. The existence of $M$ and $K$ is just the Doob-Meyer decomposition of supermartingales of class [D] (see [4, p. 221]). Besides if $R(\eta$ ) belongs to $\mathscr{S}^{2}$, then the process $K$ does. This is a direct consequence of the dual predictable projection of $K$ (see [4, p. 221]). The proof of $\left\{\Delta K^{d}>0\right\} \subset$ $\left\{R(\eta)_{-}=\eta_{-}\right\}$is given in $[5$, p. 131]. Finally since the filtration is generated by a Brownian motion and an independent Poisson measure, the jumps of $M$ occur only at inaccessible stopping times. Therefore when $K^{d}$ jumps, which is a predictable process, the process $R(\eta)$ has the same jump. It follows that $\Delta_{t} K^{d}=\left(R(\eta)_{t-}-R(\eta)_{t}\right)^{+} 1_{\left[R(\eta)_{t-}=\eta_{t-}\right]}=\left(\eta_{t-}-R(\eta)_{t}\right)^{+} 1_{\left[R(\eta)_{t-}=\eta_{t-}\right]}$. The proof of property (A2) is complete.

Let $X:=\left(X_{t}\right)_{t \leqslant 1}$ be a process of class [D]. The predictable projection of $X$, which we denote by $X^{p}$, is an $\mathscr{F}_{t}$-predictable process which satisfies $\mathbf{E}\left[X_{\tau} \mid \mathscr{F}_{\tau-}\right]=X_{\tau}^{p}$ for any $\mathscr{F}_{t}$-predictable stopping time. The process $X$ is called regular if it satisfies $X_{t}^{p}=X_{t-}$, for any $t \leqslant 1$.

The following result is related to existence of an optimal stopping time when the Snell envelope is regular.

(A3) Let $\eta$ be a process of $\mathscr{S}^{2}$ and $R(\eta)$ its Snell envelope whose decomposition is $M-K$. For $t \leqslant 1$, let $\tau_{t}$ be the stopping time defined as follows:

$$
\tau_{t}=\inf \left\{s \geqslant t: K_{s}-K_{t}>0\right\} \wedge 1 \text {. }
$$

If $R(\eta)$ is regular, i.e., $R(\eta)^{p}=R(\eta)_{-}$, then $K^{d} \equiv 0$ and $\tau_{t}$ is optimal in $\mathscr{T}_{t}$, i.e., it satisfies:

(i) $\mathbf{E}\left[\eta_{\tau_{t}}\right]=\sup _{\tau \geqslant t} \mathbf{E}\left[\eta_{\tau}\right]$;

(ii) $R(\eta)_{\tau_{t}}=\eta_{\tau_{t}}$ and $\left(R(\eta)_{s \wedge \tau_{t}}\right)_{s \geqslant t}$ is an $\mathscr{F}_{s}$-martingale.

A word about the proofs of those claims. The continuity of $K$ when $R(\eta)$ is regular is stated in [4, p. 214]. As for the optimality of $\tau_{t}$, one can see, e.g., [5, p. 140]. 
Acknowledgements. This paper has been carried out when the second author visited Université du Maine (Le Mans, France). Their hospitality was greatly appreciated. We would like also to thank the anonymous referee whose suggestions led to the improvement of the manuscript.

\section{REFERENCES}

1. Barles G., Buckdahn R., Pardoux E. Backward stochastic differential equations and integral-partial differential equations. - Stochastics and Stochastics Rep. 1997, v. 60, № $1-2$, p. $57-83$.

2. Bielecki T., Crepey S., Jeanblanc M., Rutkowski M. Defautable options in a Markovian intensity model of credit risk. - Math. Finance, 2008, v. 18, № 4, p. 493-518.

3. Cvitanic J., Karatzas I. Backward stochastic differential equations with reflection and Dynkin games. - Ann. Probab., 1996, v. 24, № 4, p. 2024-2056.

4. Dellacherie C., Meyer P. A. Probabilités et potentiel. Chap. V-VIII. Paris: Hermann, 1980, $476 \mathrm{p}$.

5. El Karoui $N$. Les aspects probabilistes du contrôle stochastique. - Lecture Notes in Math., 1981, v. 876, p. 73-238.

6. El Karoui N., Kapoudjian C., Pardoux E., Peng S., Quenez M. C. Reflected solutions of backward SDE's and related obstacle problems for PDE's. - Ann. Probab., 1997, v. 25 , № 2 , p. $702-737$.

7. El Karoui N., Pardoux E., Quenez M.-C. Reflected backward SDEs and American options. - Numerical Methods in Finance. Ed. by L. Rogers and D. Talay. Cambridge: Cambridge Univ. Press, 1997, p. 215-231. (Publ. Newton Inst., v. 13.)

8. Hamadène $S$. Mixed zero-sum differential game and American game options. — SIAM J. Control Optim., 2006, v. 45, № 2, p. 496-518.

9. Hamadène S. Reflected BSDE's with discontinuous barriers and application. Stochastics Stochastics Rep., 2002, v. 74, № 3-4, p. 571-596.

10. Hamadène S., Lepeltier J. P. Reflected BSDEs and mixed game problems. — Stochastic Process. Appl., 2000, v. 85, № 2, p. 177-188.

11. Hamadène S., Ouknine $Y$. Reflected backward stochastic differential equation with jumps and random obstacle. - Electron. J. Probab., 2003, v. 8, № 2, p. 1-20.

12. Ватанабэ C., Икэда Н. Стохастические дифференциальные уравнения и диффузионные процессы. М.: Наука, 1986, 445 с.

13. Lepeltier J. P., Xu M. Penalization method for reflected backward stochastic differential equations with one r.c.l.l. barrier. - Statist. Probab. Lett., 2005, v. 75, № 1, p. $58-66$.

14. Tang S. J., Li X.J. Necessary conditions for optimal control of stochastic systems with random jumps. - SIAM J. Control Optim., 1994, v. 33, № 5, p. 1447-1475.

15. Peng $S$., $X u M$. The smallest $g$-supermartingale and reflected BSDE with single and double $L^{2}$ obstacles. - Ann. Inst. H. Poincaré, 2005, v. 41, № 3, p. 605-630.

16. Pardoux E., Peng S. Adapted solutions of a backward stochastic differential equation. - Systems Control Lett., 1990, v. 14, p. 51-61.

17. Pardoux E. BSDEs, weak convergence and homogenization of semilinear PDEs. Nonlinear Analysis, Differential Equations and Control. Ed. by F. Clarke and R. Stern. Dordrecht: Kluwer, 1999, p. 503-549.

18. Pardoux E., Peng S. Backward stochastic differential equations and quasilinear parabolic partial differential equations. - Lecture Notes in Control Inform. Sci., 1992 , v. 176, p. $200-217$.

19. Peng S. Monotonic limit theorem of BSDE and nonlinear decomposition theorem of Doob-Meyer's type. — Probab. Theory Related Fields, 1999, v. 113, № 4, p. 473-499. 